\title{
Analytical Approach to Differential Equations with Piecewise Continuous Arguments via Modified Piecewise Variational Iteration Method
}

\author{
Qi Wang \\ School of Applied Mathematics, Guangdong University of Technology, Guangzhou, China \\ Email: bmwzwq@126.com
}

Received October 2013

\begin{abstract}
In the present article, we apply the modified piecewise variational iteration method to obtain the approximate analytical solutions of the differential equations with piecewise continuous arguments. This technique provides a sequence of functions which converges to the exact solution of the problem. Moreover, this method reduces the volume of calculations because it does not need discretization of the variables, linearization or small perturbations. The results seem to show that the method is very reliable and convenient for solving such equations.
\end{abstract}

\section{KEYWORDS}

Delay Differential Equations; Piecewise Continuous Arguments; Variational Iteration Method; Approximation

\section{Introduction}

Differential equations with piecewise continuous arguments (EPCA) are special type of delay differential equations (DDEs). The theory of EPCA was initiated in [1,2] and developed by many authors [3-7]. These systems have been under intensive investigation for the last twenty years. EPCA describe hybrid dynamical systems and combine properties of both differential and difference equations. They are appeared in modeling of various problems in real life such as biology, mechanics, and electronics. For some applications of this equation we refer the interested reader to [1,8-10]. Several important properties of the analytic solution of EPCA as well as numerical methods have been studied in [11-16].

In this paper, we consider the following two EPCA:

$$
\left\{\begin{array}{l}
\left.u^{\prime}(t)=a_{0} u(t)+a_{1} u(t t]\right), t \geq 0, \\
u(0)=u_{0},
\end{array}\right.
$$

and the coupled system

$$
\left\{\begin{array}{l}
x^{\prime}(t)=a_{2} x(t)+a_{3} y([t]) \\
y^{\prime}(t)=a_{4} y(t)+a_{5} x([t])
\end{array}\right.
$$

with initial value $X(0)=\left(x_{0}, y_{0}\right)^{T}$, where $a_{i} \quad(i=0,1, \cdots, 5)$ are real constants and [.] denotes the greatest integer function and $X(t)=(x(t), y(t))^{T}$.

In this work, we apply the modified piecewise variational iteration method (MPVIM) to systems (1) and (2) to obtain approximate analytical solutions. The VIM gives several successive approximations by using the iteration of the correction functional. This method was proposed by the Chinese researcher Jihuan He [17-19] as a modification of a general Lagrange multiplier method [20]. VIM is one of the non-perturbation methods that does not require any small or large parameter. An elementary introduction of VIM is given in [21]. The main concepts in VIM, such as general Lagrange multiplier, restricted variation, correction functional are explained systemically. For more comprehensive survey on this method and its applications, the reader is referred to the review articles [22,23] and the references therein. 
The VIM has been favorably applied to various kinds of linear and nonlinear problems. The main property of the method is in flexibility and ability to solve linear and nonlinear equations accurately and conveniently. The flexibility and adaptation provided by this method have made the method a strong candidate for approximate analytical solutions. The VIM plays an important role in recent researches for solving various kinds of problems (see for example [24-28] and the references therein). However, the researches on the application of VIM on DDE are relatively fewer. As far as we know, only delay Burgers equation [29], delay logistic equation [30] and pantograph equation [31-33] are considered. As for the analytical study of EPCA with VIM, up to now, there are almost no results published. Therefore, we will conduct this study.

The organization of this paper is as follows. In Section 2, we simply provide the mathematical framework of the VIM. In Section 3, we apply the modified piecewise variational iteration method on the systems (1) and (2) after analyzing the conventional VIM and piecewise variational iteration method. Some numerical results are given in Section 4. Finally, in Section 5, a brief conclusion is provided.

\section{He's Variational Iteration Method}

In this section, we introduce the basic idea underlying the VIM for solving nonlinear equations. Consider the general differential equation

$$
L u+N u=g(x),
$$

where $L$ and $N$ are linear and nonlinear operators, respectively, and $g(x)$ is the inhomogeneous term. In VIM, a correction functional for (3) can be written as

$$
u_{n+1}(x)=u_{n}(x)+\int_{0}^{x} \lambda(s)\left[L u_{n}(s)+N \tilde{u}_{n}(s)-g(s)\right] d s,
$$

where $\lambda$ is a general Lagrange's multiplier, which can be identified optimally via integration by parts and the variational theory, and $\tilde{u}_{n}$ denotes the restricted variation, i.e. $\delta \tilde{u}_{n}=0$. It is to be noted that the Lagrange multiplier $\lambda$ can be a constant or a function. After determining the Lagrange multiplier $\lambda$, an iteration formula, without restricted variation, should be used for the determination of the successive approximations $u_{n+1}(x)$ of the solution $u(x)$. The zeroth approximation $u_{0}$ can be selected freely. Consequently, the solution is given by

$$
u(x)=\lim _{n \rightarrow \infty} u_{n}(x)
$$

\section{The Application of VIM}

In this section the application of VIM is discussed for solving systems (1) and (2).

\subsection{System (1)}

We consider system (1), according to the VIM, the correction function is given by

$$
u_{n+1}(t)=u_{n}(t)+\int_{0}^{t} \lambda(s)\left(u_{n}^{\prime}(s)-a \bar{u}_{n}(s)-a_{1} \bar{u}_{n}([s])\right) d s
$$

To find the optimal value of $\lambda$ we have

$$
\delta u_{n+1}(t)=\delta u_{n}(t)+\delta \int_{0}^{t} \lambda(s) u_{n}^{\prime}(s) d s,
$$

that results

$$
\delta u_{n+1}(t)=\left(1+\left.\lambda\right|_{s=t}\right) \delta u_{n}(t)-\delta \int_{0}^{t} \lambda^{\prime}(s) u_{n}(s) d s
$$

Thus we have the following stationary conditions

$$
\left\{\begin{array}{l}
1+\left.\lambda\right|_{s=t}=0 \\
\left.\lambda^{\prime}(s)\right|_{s=t}=0 .
\end{array}\right.
$$

This in turn gives $\lambda=-1$. So we obtain the following iteration formula

$$
u_{n+1}(t)=u_{n}(t)-\int_{0}^{t}\left(u_{n}^{\prime}(s)-a_{0} u_{n}(s)-a_{1} u_{n}([s])\right) d s,
$$


and the approximation solution is given by

$$
u(t)=\lim _{n \rightarrow \infty} u_{n+1}(t) .
$$

During the process of computation, the greatest integer function [.] causes us many problems. To overcome them, we recall a modified VIM: the piecewise variational iteration method (PVIM), which was introduced by Geng [34,35]. In PVIM, the interval $[0, X]$ is divided into some equal subintervals, then the $n_{i}$-order approximation $u_{i, n_{i}}(x)$ are obtained on these subintervals. Following this way, we introduce the modified piecewise variational iteration method (MPVIM). In our method, the interval $[0, \infty)$ is divided into lots of subintervals $[k, k+1)$ with unit length, where $k \in N$.

On the interval $[0,1]$, let

$$
\begin{gathered}
u_{1, n+1}(t)=u_{1,0}(t)-\int_{0}^{t}\left(u_{1, n}^{\prime}(s)-a_{0} u_{1, n}(s)-a_{1} u_{1, n}([s])\right) d s, \\
u_{1,0}(t)=u(0),
\end{gathered}
$$

where $t \in[0,1]$. Then we can obtain the $n_{1}$-order approximation $u_{1, n_{1}}(t)$ on $[0,1]$.

On the interval $[1,2]$, let

$$
\begin{gathered}
u_{2, n+1}(t)=u_{2,0}(t)-\int_{0}^{t}\left(u_{2, n}^{\prime}(s)-a_{0} u_{2, n}(s)-a_{1} u_{2, n}([s])\right) d s, \\
u_{2,0}(t)=u_{1, n_{1}}(1) .
\end{gathered}
$$

The integration in (13) can be computed in $[0,1]$ and $[1, t]$, respectively. Then the $n_{2}$-order approximation $u_{2, n_{2}}(t)$ on $[1,2]$ can be obtained.

In a similar way, on the interval $[k-1, k], k=3,4, \cdots$ let

$$
\begin{gathered}
u_{k, n+1}(t)=u_{k, 0}(t)-\int_{0}^{t}\left(u_{k, n}^{\prime}(s)-a_{0} u_{k, n}(s)-a_{1} u_{k, n}([s])\right) d s, \\
u_{k, 0}(t)=u_{k-1, n_{k-1}}(k-1) .
\end{gathered}
$$

The integration in (14) can be computed in a series of subintervals: $[0,1],[1,2], \cdots,[k-1, t]$. Then we can obtain the $n_{k}$-order approximation $u_{k, n_{k}}(t)$ on $[k-1, k]$.

Therefore, according to (12)-(14), the approximation of (1) on the entire interval $[0, \infty)$ can be obtained.

\subsection{System (2)}

According to VIM, the iteration formula for (2) can be constructed as follows

$$
\begin{aligned}
& x_{n+1}(t)=x_{n}(t)-\int_{0}^{t}\left(x_{n}^{\prime}(s)-a_{2} x_{n}(s)-a_{3} y_{n}([s])\right) d s, \\
& y_{n+1}(t)=y_{n}(t)-\int_{0}^{t}\left(y_{n}^{\prime}(s)-a_{4} y_{n}(s)-a_{5} x_{n}([s])\right) d s .
\end{aligned}
$$

Similar to Subsection 3.1, in view of MPVIM we have the following formulas.

On the interval $[0,1]$, let

$$
\begin{gathered}
x_{1, n+1}(t)=x_{1,0}(t)-\int_{0}^{t}\left(x_{1, n}^{\prime}(s)-a_{2} x_{1, n}(s)-a_{3} y_{1, n}([s])\right) d s, \\
y_{1, n+1}(t)=y_{1,0}(t)-\int_{0}^{t}\left(y_{1, n}^{\prime}(s)-a_{4} y_{1, n}(s)-a_{5} x_{1, n}([s])\right) d s, \\
x_{1,0}(t)=x(0), \\
y_{1,0}(t)=y(0) .
\end{gathered}
$$

Then we can obtain the $n_{1}$-order approximation $X_{1, n_{1}}(t)$ on $[0,1]$, where $X(t)=(x(t), y(t))^{T}$. On the interval $[1,2]$, let

$$
\begin{aligned}
& x_{2, n+1}(t)=x_{2,0}(t)-\int_{0}^{t}\left(x_{2, n}^{\prime}(s)-a_{2} x_{2, n}(s)-a_{3} y_{2, n}([s])\right) d s, \\
& y_{2, n+1}(t)=y_{2,0}(t)-\int_{0}^{t}\left(y_{2, n}^{\prime}(s)-a_{4} y_{2, n}(s)-a_{5} x_{2, n}([s])\right) d s,
\end{aligned}
$$




$$
\begin{aligned}
& x_{2,0}(t)=x_{1, n_{1}}(1), \\
& y_{2,0}(t)=y_{1, n_{1}}(1) .
\end{aligned}
$$

Then we can obtain the $n_{2}$-order approximation $X_{2, n_{2}}(t)$ on $[1,2]$.

Similarly, on the interval $[k-1, k], k=3,4, \cdots$ let

$$
\begin{gathered}
x_{k, n+1}(t)=x_{k, 0}(t)-\int_{0}^{t}\left(x_{k, n}^{\prime}(s)-a_{2} x_{k, n}(s)-a_{3} y_{k, n}([s])\right) d s, \\
y_{k, n+1}(t)=y_{k, 0}(t)-\int_{0}^{t}\left(y_{k, n}^{\prime}(s)-a_{4} y_{k, n}(s)-a_{5} x_{k, n}([s])\right) d s, \\
x_{k, 0}(t)=x_{k-1, n_{k-1}}(k-1), \\
y_{k, 0}(t)=y_{k-1, n_{k-1}}(k-1) .
\end{gathered}
$$

Then we can obtain the $n_{k}$-order approximation $X_{k, n_{k}}(t)$ on $[k-1, k]$.

Therefore, according to (16)-(18), the approximation of coupled system (2) on the entire interval $[0, \infty)$ can be obtained.

\section{Results and Discussion}

In this section, we apply the MPVIM presented in Section 3 and the classical $\theta$-methods to two concrete EPCA. Numerical results show that the MPVIM is very effective.

For (1), we choose $a_{0}=2, a_{1}=-1$ and $u_{0}=1$. According to (12)-(14), taking $k=3$ and $n_{i}=5$, $i=1, \cdots, k$. We can obtain the approximations of $(1)$ on $[0,3]$. The numerical results are depicted in Figure 1 . This figure shows the comparison of approximation obtained by using the present method with the exact solution and the numerical solution. Moreover, for (2), we choose $a_{2}=1, a_{3}=-2, a_{4}=2, a_{5}=-1$ and $x_{0}=y_{0}=1$. In Figure 2 we compare the 5th-order approximation of MPVIM with the numerical solution.
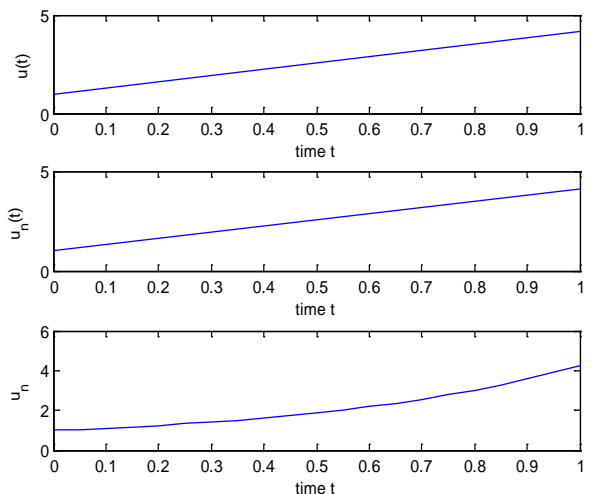

Figure 1. A comparison of the results of the exact solution (upper), the 5th-order MPVIM solution (middle) and the numerical solution (lower) with $\theta=0.6$ and $m=20$ to (1).
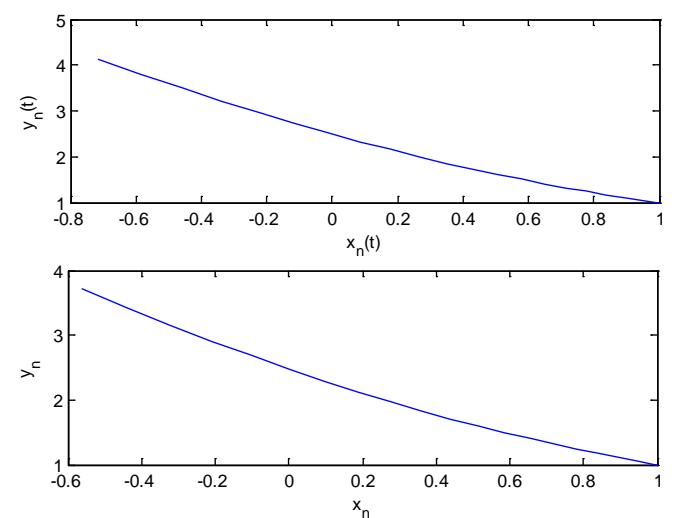

Figure 2. A comparison of the results of the 5th-order MPVIM solution (upper) and the numerical solution (lower) with $\theta=0.3$ and $m=20$ to (2). 
The above numerical examples demonstrate that the present method is quite effective and simple.

\section{Conclusions}

An efficient algorithm based on the VIM has been successfully applied to the EPCA. As can be seen from the numerical results, implementing only a few steps in the MPVIM, the approximate analytical solutions with high accuracy can be obtained.

It can be concluded that the MPVIM is a powerful and promising tool for solving such kinds of delay differential equations. This method can also be extended to the EPCA of the advanced type and mixed type, which are our future research issues.

\section{Acknowledgements}

The author would like to thank the reviewers for his/her hard work. In addition, we thank Professor Fazhan Geng for his helpful assistance.

\section{REFERENCES}

[1] S. Busenberg and K. L. Cooke, "Models of Vertically Transmitted Diseases with Sequential-Continuous Dynamics, Nonlinear Phenomena in Mathematical Sciences,” In: V. Lakshmikantham, Ed., Academic Press, New York, 1982, pp. 179-187.

[2] K. L. Cooke and J. Wiener, "Retarded Differential Equations with Piecewise Constant Delays," Journal of Mathematical Analysis and Applications, Vol. 99, 1984, pp. 265-297. http://dx.doi.org/10.1016/0022-247X(84)90248-8

[3] Y. K. Huang, “A Nonlinear Equation with Piecewise Constant Argument,” Applied Analysis, Vol. 33, 1989, pp. $183-190$. http://dx.doi.org/10.1080/00036818908839871

[4] J. L. Hong, R. Obayab and A. Sanzb, “Almost Periodic Type Solutions of Some Differential Equations with Piecewise Constant Argument,” Nonlinear Analysis, Vol. 45, 2001, pp. 661-688. http://dx.doi.org/10.1016/S0362-546X(98)00296-X

[5] L. Dai and M. C. Singh, "A New Approach with Piecewise Constant Arguments to Approximate and Numerical Solutions of Oscillatory Problems,” Journal of Sound and Vibration, Vol. 263, 2003, pp. 535-548. http://dx.doi.org/10.1016/S0022-460X(02)01065-9

[6] G. Papaschinopoulos, G. Stefanidou and P. Efraimidis, "Existence, Uniqueness and Asymptotic Behavior of the Solutions of a Fuzzy Differential Equation with Piecewise Constant Argument,” Information Science, Vol. 177, 2007, pp. 3855-3870. http://dx.doi.org/10.1016/j.ins.2007.03.006

[7] M. Pinto, “Asymptotic Equivalence of Nonlinear and Quasi Linear Differential Equations with Piecewise Constant Arguments,” Mathematical and Computer Modelling, Vol. 49, 2009, pp. 1750-1758. http://dx.doi.org/10.1016/j.mcm.2008.10.001

[8] L. Dai and M. C. Singh, “On Oscillatory Motion of Spring-mass Systems Subjected to Piecewise Constant Forces,” Journal of Sound and Vibration, Vol. 173, 1994, pp. 217-232. http://dx.doi.org/10.1006/jsvi.1994.1227

[9] N. M. Murad and A. Celeste, "Linear and Nonlinear Characterization of Loading Systems Under Piecewise Discontinuous Disturbances Voltage: Analytical and Numerical Approaches,” Proceedings of International Conference on Power Electronics Systems and Applications, November 2004, pp. 291-297.

[10] J. Wiener and V. Lakshmikantham, “A Damped Oscillator with Piecewise Constant Time Delay,” Nonlinear Studies, Vol. 1, 2000, pp. 78-84.

[11] R. Yuan, “On Favard’s Theorems,” Journal of Differential Equations, Vol. 249, 2010, pp. 1884-1916. http://dx.doi.org/10.1016/j.jde.2010.07.014

[12] E. Ait Dads and L. Lhachimi, "Pseudo Almost Periodic Solutions for Equation with Piecewise Constant Argument," Journal of Computational and Applied Mathematics, Vol. 371, 2010, pp. 842-854. http://dx.doi.org/10.1016/j.jmaa.2010.06.032

[13] M. U. Akhmet, D. Arugaslanc and E. Y1lmaz, "Method of Lyapunov Functions for Differential Equations with Piecewise Constant Delay,” Journal of Computational and Applied Mathematics, Vol. 235, 2011, pp. 4554-4560. http://dx.doi.org/10.1016/j.cam.2010.02.043

[14] M. Z. Liu, M. H. Song and Z. W. Yang, "Stability of Runge-Kutta Methods in the Numerical Solution of Equation $\mathrm{u}^{\prime}(\mathrm{t})=\mathrm{au}(\mathrm{t})+\mathrm{a}_{0} \mathrm{u}([\mathrm{t}])$,” Journal of Computational and Applied Mathematics, Vol. 166, 2004, pp. 361-370. http://dx.doi.org/10.1016/j.cam.2003.04.002

[15] W. J. Lv, Z. W. Yang and M. Z. Liu, "Stability of Runge-Kutta Methods for the Alternately Advanced and Retarded Differential Equations with Piecewise Continuous Arguments,” Computers \& Mathematics with Applications, Vol. 54, 2007, pp. 326335. http://dx.doi.org/10.1016/j.camwa.2006.07.018

[16] M. Z. Liu, J. F. Gao and Z. W. Yang, "Preservation of Oscillations of the Runge-Kutta Method for Equation $\mathrm{x}^{\prime}(\mathrm{t})+\mathrm{ax}(\mathrm{t})+\mathrm{a}_{1} \mathrm{x}([\mathrm{t}-1])=0$,” Computers \& Mathematics with Applications, Vol. 58, 2009, pp. 1113-1125. 
http://dx.doi.org/10.1016/j.camwa.2009.07.030

[17] J. H. He, "Variational Iteration Method for Delay Differential Equations," Communications in Nonlinear Science and Numerical Simulation, Vol. 2, 1997, pp. 235-236. http://dx.doi.org/10.1016/S1007-5704(97)90008-3

[18] J. H. He, “Approximate Solution of Nonlinear Differential Equations with Convolution Product Non-Linearities,” Computer Methods in Applied Mechanics and Engineering, Vol. 167, 1998, pp. 69-73. http://dx.doi.org/10.1016/S0045-7825(98)00109-1

[19] J. H. He, "Variational Iteration Method for Autonomous Ordinary Differential Systems,” Applied Mathematics and Computation, Vol. 114, 2000, pp. 115-123. http://dx.doi.org/10.1016/S0096-3003(99)00104-6

[20] M. Inokuti, H. Sekine and T. Mura, “General Use of the Lagrange Multiplier in Non-linear Mathematical Physics,” In: Variational Methods in the Mechanics of Solids, Pergamon Press, Oxford, 1978, pp. 156-162.

[21] J. H. He, "Variational Iteration Method-Some Recent Results and New Interpretation,” Journal of Computational and Applied Mathematics, Vol. 207, 2007, pp. 3-17. http://dx.doi.org/10.1016/j.cam.2006.07.009

[22] J. H. He and X. H. Wu, "Variational Iteration Method: New Development and Applications," Computers \& Mathematics with Applications, Vol. 54, 2007, pp. 881-894. http://dx.doi.org/10.1016/j.camwa.2006.12.083

[23] J. H. He, G. C. Wu and F. Austin, “The Variational Iteration Method Which Should Be Followed,” Nonlinear Science Letters A, Vol. 1, 2010, pp. 1-30.

[24] G. C. Wu, “A Fractional Variational Iteration Method for Solving Fractional Nonlinear Differential Equations,” Computers \& Mathematics with Applications, Vol. 61, 2011, pp. 2186-2190. http://dx.doi.org/10.1016/j.camwa.2010.09.010

[25] J. F. Lu, “An Analytical Approach to the Fornberg-Whitham Type Equations by Using the Variational Iteration Method,” Computers \& Mathematics with Applications, Vol. 61, 2011, pp. 2010-2013. http://dx.doi.org/10.1016/j.camwa.2010.08.052

[26] M. M. Khader, "Numerical and Theoretical Treatment for Solving Linear and Nonlinear Delay Differential Equations Using Variational Iteration Method,” Arab Journal of Mathematical Sciences, Vol. 19, No. 2, 2013, pp. 243-256. http://dx.doi.org/10.1016/j.ajmsc.2012.09.004

[27] G. Yang and R. Y. Chen, “Choice of an Optimal Initial Solution for a Wave Equation in the Variational Iteration Method,” Computers \& Mathematics with Applications, Vol. 61, 2011, pp. 2053-2057. http://dx.doi.org/10.1016/j.camwa.2010.08.068

[28] A. F. Elsayed, "Comparison between Variational Iteration Method and Homotopy Perturbation Method for Thermal Diffusion and Diffusion Thermo Effects of Thixotropic Fluid through Biological Tissues with Laser Radiation Existence,” Applied Mathematical Modelling, Vol. 37, no.6, 2013, pp. 3660-3673. http://dx.doi.org/10.1016/j.apm.2012.07.016

[29] E. S. Fahmy, H. A. Abdusalam and K. R. Raslan, “On the Solutions of the Time-delayed Burgers Equation,” Nonlinear Analysis, Vol. 69, 2008, pp. 4775-4786. http://dx.doi.org/10.1016/j.na.2007.11.027

[30] M. Dehghan and R. Salehi, "Solution of a Nonlinear Time-delay Model in Biology via Semi-analytical Approaches," Computer Physics Communications, Vol. 181, 2010, pp. 1255-1265. http://dx.doi.org/10.1016/j.cpc.2010.03.014

[31] Z. H. Yu, "Variational Iteration Method for Solving the Multi-Pantograph Delay Equation,” Physical Letter A, Vol. 372, 2008, pp. 6475-6479. http://dx.doi.org/10.1016/j.physleta.2008.09.013

[32] A. Saadatmandi and M. Dehghan, "Variational Iteration Method for Solving a Generalized Pantograph Equation,” Computers \& Mathematics with Applications, Vol. 58, 2009, pp. 2190-2196. http://dx.doi.org/10.1016/j.camwa.2009.03.017

[33] X. M. Chen and L. J. Wang, "The Variational Iteration Method for Solving a Neutral Functional-Differential Equation with Proportional Delays,” Computers \& Mathematics with Applications, Vol. 59, 2010, pp. 2696-2702. http://dx.doi.org/10.1016/j.camwa.2010.01.037

[34] F. Z. Geng, Y. Z. Lin and M. G. Cui, “A Piecewise Variational Iteration Method for Riccati Differential Equations,” Computers \& Mathematics with Applications, Vol. 58, 2009, pp. 2518-2522. http://dx.doi.org/10.1016/j.camwa.2009.03.063

[35] F. Z. Geng, “A Piecewise Variational Iteration Method for Treating a Nonlinear Oscillator of a Mass Attached to a Stretched Elastic Wire,” Computers \& Mathematics with Applications, Vol. 62, 2011, pp. 1641-1644. http://dx.doi.org/10.1016/j.camwa.2011.05.004 